Proyecciones Journal of Mathematics

Vol. 36, No 2, pp. 283-298, June 2017.

Universidad Católica del Norte

Antofagasta - Chile

\title{
Some new classes of vertex-mean graphs
}

\author{
A. Lourdusamy \\ St. Xavier's College (Autonomous), India \\ and \\ M. Seenivasan \\ Sri Paramakalyani College, India \\ Received: April 2016. Accepted: January 2017
}

\begin{abstract}
A vertex-mean labeling of a $(p, q)$ graph $G=(V, E)$ is defined as an injective function $f: E \longrightarrow\left\{0,1,2, \ldots, q_{*}\right\}, q_{*}=\max (p, q)$ such that the function $f^{V}: V \longrightarrow \mathbf{N}$ defined by the rule $f^{V}(v)=$ Round $\left(\frac{\sum_{e \in E_{v}} f(e)}{d(v)}\right)$ satisfies the property that

$f^{V}(V)=\left\{f^{V}(u): u \in V\right\}=\{1,2, \ldots, p\}$, where $E_{v}$ denotes the set of edges in $G$ that are incident at $v, \mathbf{N}$ denotes the set of all natural numbers and Round is the nearest integer function. A graph that has a vertex-mean labeling is called vertex-mean graph or $V$-mean graph. In this paper, we study $V$-mean behaviour of certain new classes of graphs and present a method to construct disconnected $V$-mean graphs.
\end{abstract}

MSC (2010) : Primary: 05C78; Secondary: 52C30.

Keywords : Mean labeling, edge labeling, vertex-mean labeling, vertexmean graphs. 


\section{Introduction}

A vertex labeling of a graph $G$ is an assignment $f$ of labels to the vertices of $G$ that induces a label for each edge $x y$ depending on the vertex labels. An edge labeling of a graph $G$ is an assignment $f$ of labels to the edges of $G$ that induces a label for each vertex $v$ depending on the labels of the edges incident on it. Vertex labelings such as graceful labeling, harmonious labelingand mean labeling and edge labelings such as edge-magic labeling, (a,d)-anti magic labeling and vertex-graceful labeling are some of the interesting labelings found in the dynamic survey of graph labeling by Gallian[2]. In fact Acharya and Germina [1] has introduced vertex-graceful graphs, as an edge-analogue of graceful graphs.

A mean labeling $f$ is an injective function from $V$ to the set $\{0,1,2, \ldots, q\}$ such that the set of edge labels defined by the rule $\left[\frac{f(u)+f(v)}{2}\right]$ for each edge $u v$ is $\{1,2, \ldots, q\}$. The mean labeling was introduced by Somasundaram and Ponraj [4]. Observe that, in a variety of practical problems, the arithmetic mean, $X$, of a finite set of real numbers $\left\{x_{1}, x_{2}, \ldots, x_{n}\right\}$ serves as a better estimate for it, in the sense that $\sum\left(x_{i}-X\right)$ is zero and $\sum\left(x_{i}-X\right)^{2}$ is the minimum. If it is required to use a single integer in the place of $X$ then $\operatorname{Round}(X)$ does this best, in the sense that $\sum\left(x_{i}-\operatorname{Round}(X)\right)$ and $\sum\left(x_{i}-\right.$ $\operatorname{Round}(X))^{2}$ are minimum, where $\operatorname{Round}(Y)$, nearest integer function of a real number, gives the integer closest to $Y$; to avoid ambiguity, it is defined to be the nearest integer greater than $Y$ if the fraction of $\mathrm{y}$ is 0.5 . Motivated by this and the concept of vertex-graceful graphs, Lourdusamy and Seenivasan [3] introduced vertex-mean labeling as an edge analogue of mean labeling as follows:

A vertex-mean labeling of a $(p, q)$ graph $G=(V, E)$ is defined as an injective function $f: E \longrightarrow\left\{0,1,2, \ldots, q_{*}\right\}, q_{*}=\max (p, q)$ such that the function $f^{V}: V \longrightarrow \mathbf{N}$ defined by the rule $f^{V}(v)=\operatorname{Round}\left(\frac{\sum_{e \in E_{v}} f(e)}{d(v)}\right)$

satisfies the property that $f^{V}(V)=\left\{f^{V}(u): u \in V\right\}=\{1,2, \ldots, p\}$, where $E_{v}$ denotes the set of edges in $G$ that are incident at $v$ and $\mathbf{N}$ denotes the set of all natural numbers. A graph that has a vertex-mean labeling is called vertex-mean graph or $V$-mean graph. They, obtained necessary conditions for a graph to be a $V$-mean graph, and proved that any 3-regular graph of order $2 m, m \geq 4$ is not a $V$-mean graph. They also proved that the path $P_{n}$, where $n \geq 3$ and the cycle $C_{n}$, the Corona $P_{n} \odot K_{m}^{C}$, where $n \geq 2$ and $m \geq 1$, the star graph $K_{1, n}$ if and only if $n \equiv 0(\bmod 2)$, and the crown 
$C_{n} \odot K_{1}$ are $V$-mean graphs. A dragon is a graph obtained by identifying an end point of a path $P_{m}$ with a vertex of the cycle $C_{n}$ and $m P_{n}$ denotes the disjoint union of $m$ copies of the path $P_{n}$. For $3 \leq p \leq n-r, C_{n}(p, r)$ denotes the graph obtained from the cycle $C_{n}$ with consecutive vertices $v_{1}$, $v_{2}, \ldots, v_{n}$ by adding the $r$ chords $v_{1} v_{p}, v_{1} v_{p+1}, \ldots, v_{1} v_{p+r-1}$. In this paper we present the $\mathrm{V}$-mean labeling of the following graphs:

1. The graph $S\left(K_{1, n}\right)$, obtained by subdividing every edge of $K_{1, n}$,

2. Dragon graph,

3. The graph obtained by identifying one vertex of the cycle $C_{3}$ with the central vertex of $K_{1, n}$,

4. The graph $C_{n}(3,1)$,

5. The graph obtained from the two cycles $C_{n}$ and $C_{m}$ by adding a new edge joining a vertex of $C_{n}$ and $C_{m}$ where $m \in\{n, n+1, n+2\}$,

6. The graph $C_{n} \cup C_{m}$,

7. The graph obtained by identifying one vertex of the cycle $C_{m}$ with a vertex of $C_{n}$ when $m=3$ or 4 .

We also explain a method to obtain disconnected V-mean graphs from $\mathrm{V}$-mean graphs. Following are some of the graphs so obtained: the graph $\bigcup_{i=1}^{k} P_{n_{i}}$, where $n_{i} \geq 3$, the graph $m P_{n}$ where $m \geq 1$ and $n \geq 3$, the graph $C_{n} \cup\left(\cup_{i=1}^{k} P_{n_{i}}\right)$, where $n_{i} \geq 3$, the graph $C_{n} \cup k P_{m}$ where $m \geq 3$, the graph $C_{n} \cup C_{m} \cup\left(\bigcup_{i=1}^{k} P_{n_{i}}\right)$, where $n_{i} \geq 3$, the graph $C_{n} \cup C_{m} \cup k P_{t}$ where $t \geq 3$.

\section{New classes of $\mathrm{V}$-mean graphs}

Theorem 2.1. The graph $S\left(K_{1, n}\right)$, obtained by subdividing every edge of $K_{1, n}$, exactly once, is a $V$-mean graph.

Proof. Let $V=\left\{u, v_{i}, w_{i}: 1 \leq i \leq n\right\}$ and $E=\left\{u v_{i}, v_{i} w_{i}: 1 \leq i \leq n\right\}$ be the vertex set and edge set of $S\left(K_{1, n}\right)$ respectively. Then $G$ has order $2 n+1$ and size $2 n$. 

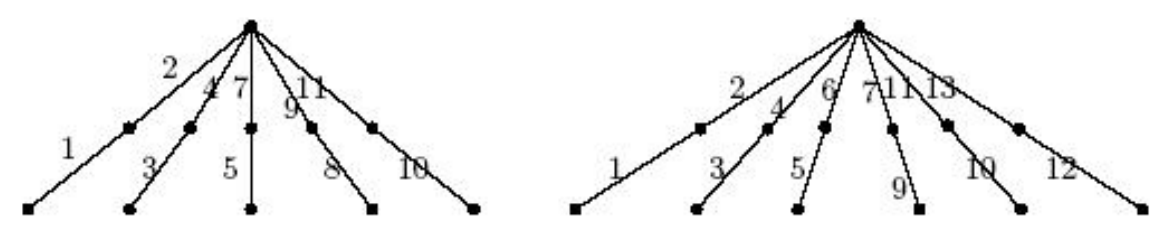

FIGURE 1. $V$-mean labeling of $S\left(K_{1,5}\right)$ and $S\left(K_{1,6}\right)$

case 1: $n$ is odd.

Let $n=2 m+1$. Define $f: E \longrightarrow\{0,1,2, \ldots, 4 m+3\}$ as follows:

$$
\begin{gathered}
f\left(u v_{i}\right)=\left\{\begin{array}{ll}
2 i & \text { if } \quad 1 \leq i \leq m \\
2 i+1 & \text { if } \quad m+1 \leq i \leq n
\end{array},\right. \text { and } \\
f\left(u_{i} v_{i}\right)=\left\{\begin{array}{cl}
2 i-1 & \text { if } 1 \leq i \leq m+1 \\
2 i & \text { if } \quad m+2 \leq i \leq n
\end{array}\right.
\end{gathered}
$$

Then it is easy to verify that

$$
\begin{aligned}
& f^{V}(u)=2 m+3, \\
& f^{V}\left(u_{i}\right)=\left\{\begin{array}{lll}
2 i & \text { if } & 1 \leq i \leq m+1 \\
2 i+1 & \text { if } & m+2 \leq i \leq n
\end{array},\right. \text { and } \\
& f^{V}\left(w_{i}\right)=\left\{\begin{array}{lll}
2 i-1 & \text { if } & 1 \leq i \leq m+1 \\
2 i & \text { if } & m+2 \leq i \leq n
\end{array}\right.
\end{aligned}
$$

Hence

$$
f^{V}(V)=\{1,2,3, \ldots, 4 m+3\}
$$

case 2: $n$ is even.

Let $n=2 m$. Define $f: E \longrightarrow\{0,1,2, \ldots, 4 m+1\}$ as follows:

$$
\begin{aligned}
& f\left(u v_{i}\right)=\left\{\begin{array}{lll}
2 i & \text { if } \quad 1 \leq i \leq m \\
2 i-1 & \text { if } i=m+1 \\
2 i+1 & \text { if } \quad m+2 \leq i \leq n
\end{array},\right. \text { and } \\
& f\left(u_{i} w_{i}\right)= \begin{cases}2 i-1 & \text { if } \quad 1 \leq i \leq m \\
2 i+1 & \text { if } \quad i=m+1 \\
2 i & \text { if } \quad m+2 \leq i \leq n\end{cases}
\end{aligned}
$$

Then, it is easy to verify that 


$$
\begin{aligned}
& f^{V}(u)=2 m+1, \\
& f^{V}\left(u_{i}\right)=\left\{\begin{array}{lll}
2 i & \text { if } & 1 \leq i \leq m+1 \\
2 i+1 & \text { if } & m+2 \leq i \leq n
\end{array},\right. \text { and } \\
& f^{V}\left(w_{i}\right)=\left\{\begin{array}{lll}
2 i-1 & \text { if } & 1 \leq i \leq m+1 \\
2 i+1 & \text { if } & i=m+1 \\
2 i & \text { if } & m+2 \leq i \leq n
\end{array}\right.
\end{aligned}
$$

Hence

$$
f^{V}(V)=\{1,2,3, \ldots, 4 m+1\} .
$$

Thus, $S\left(K_{1, n}\right)$ is $V$-mean. $\square V$-mean labeling of $S\left(K_{1,5}\right)$ and $S\left(K_{1,6}\right)$ are shown in Figure 1.

Theorem 2.2. A dragon graph is $V$-mean.

Proof. Let $G$ be a dragon consisting of the path $P_{m}: v_{1} v_{2} \ldots v_{m}$ and the cycle $C_{n}: u_{1} u_{2} \ldots u_{n}$. Let $v_{m}$ be identified with $u_{n}$ and $r=\left\lceil\frac{n}{2}\right\rceil$. Let $e_{i}=v_{i} v_{i+1}, 1 \leq i \leq m-1, e_{i+1}^{\prime}=u_{i} u_{i+1}, 1 \leq i \leq n-1$ and $e_{1}^{\prime}=u_{n} u_{1}$ be the edges of $G$. Observe that $G$ has order and size both equal to $m+n-1$. The edges of $G$ are labeled as follows:

For $1 \leq i \leq m-1$, the integer $i$ is assigned to the edge $e_{i}$. The odd and even integers from 1 to $n$ are respectively arranged in increasing sequences $\alpha_{1}, \alpha_{2}, \ldots, \alpha_{r}$ and $\beta_{1}, \beta_{2}, \ldots, \beta_{n-r}$ and $m-1+\alpha_{k}$ is assigned to $e_{k}^{\prime}$ and $m-$ $1+\beta_{k}$ is assigned to $e_{n-k+1}^{\prime}$.

\begin{tabular}{|c|c|}
\hline vertex & induced edge label \\
\hline$v_{i}, 1 \leq i \leq m-1$ & $i$ \\
\hline$u_{k}, 1 \leq k \leq n-r$ & $m-1+\beta_{k}$ \\
\hline$u_{n-k+1}, 1 \leq i \leq r$ & $m-1+\alpha_{k}$ \\
\hline
\end{tabular}

Table 1. Induced vertex labels

Clearly the edges of $G$ receive distinct labels from $\{0,1,2, \ldots, m+n-1\}$ and the vertex labels induced are $1,2, \ldots, m+n-1$ as illustrated in Table 1. Thus $G$ is $V$-mean. 
For example $V$-mean labelings of dragons obtained from $P_{5}$ and $C_{7}$ and $P_{6}$ and $C_{7}$ are shown in Figure 2.

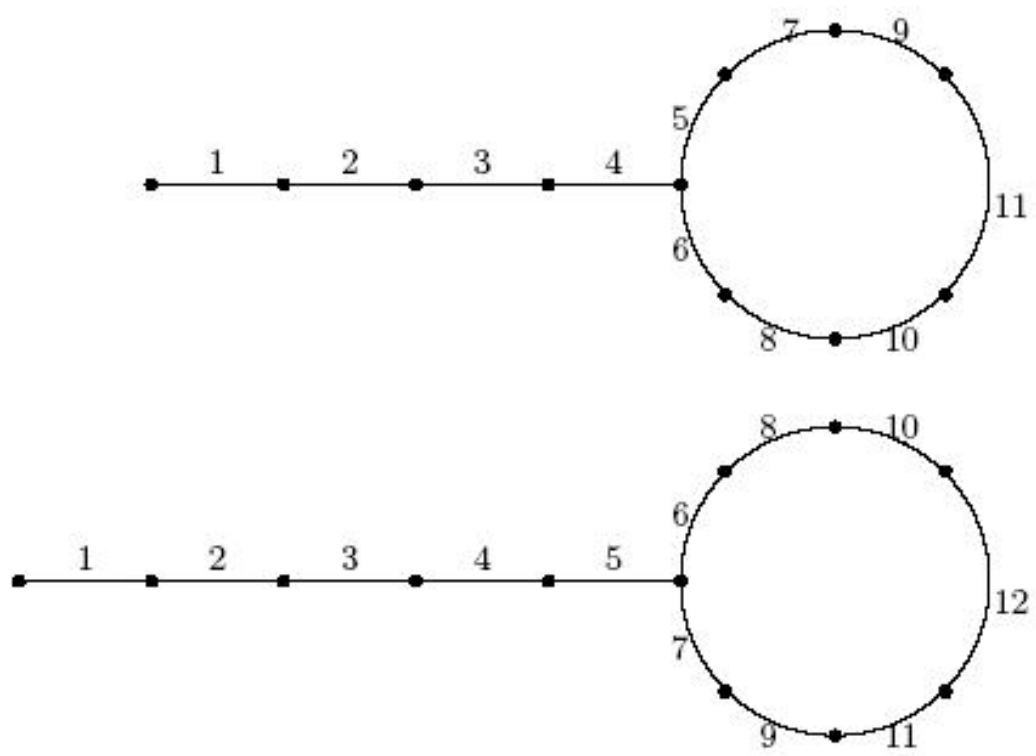

FIGURE 2. $V$-mean labelings of dragons

Theorem 2.3. Let $G$ be a graph obtained by identifying one vertex of the cycle $C_{3}$ with the central vertex of $K_{1, n}$. Then $G$ is $V$-mean.

Proof. Let $u_{1}, u_{2}, u_{3}$ be the consecutive vertices of $C_{3}$. Let $V\left(K_{1, n}\right)=$ $\left\{w, w_{1}, w_{2}, \ldots, w_{n}\right\}$ with $\operatorname{deg} w=n$ and $u_{1}$ be identified with $w$. Then $G$ is of order and size both equal to $n+3$. Let $r=\left\lfloor\frac{n}{2}\right\rfloor$. Define $f: E(G) \longrightarrow$ $\{0,1,2, \ldots, n+3\}$ as follows:

$$
\begin{gathered}
f\left(w u_{2}\right)=r+2, f\left(w u_{3}\right)=r+4, f\left(u_{2} u_{3}\right)=r+3, \text { and } \\
f\left(w w_{i}\right)= \begin{cases}i & \text { if } 1 \leq i \leq r+1 \\
i+3 & \text { if } r+2 \leq i \leq n\end{cases}
\end{gathered}
$$

Then, it follows easily that

$$
f^{V}(w)=r+2, f^{V}\left(u_{2}\right)=r+3, f^{V}\left(u_{3}\right)=r+4, \text { and }
$$




$$
f^{V}\left(w_{i}\right)=\left\{\begin{array}{ll}
i & \text { if } 1 \leq i \leq r+1 \\
i+3 & \text { if } r+2 \leq i \leq n
\end{array} .\right.
$$

Hence $f^{V}(V(G))=\{1,2,3, \ldots n+3\}$. Thus $G$ is a $V$-mean graph. $V$-mean labeling of the graphs obtained from $K_{1,5}$ and $K_{1,6}$ by idenntifying the central vertex of each with a vertex of $C_{3}$ as shown in Figure 3.
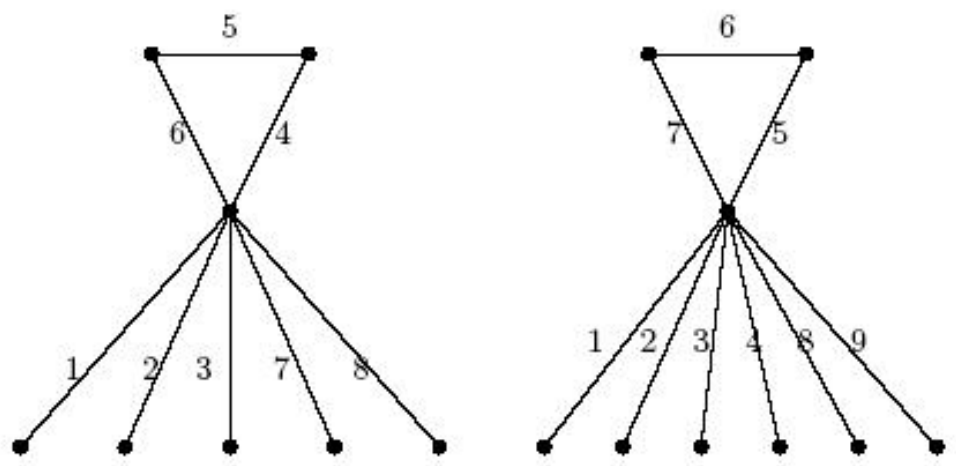

Figure 3. $V$-mean labeling of $G$ obtained from $K_{1,5}$ and $K_{1,6}$

Theorem 2.4. The graph $C_{n}(3,1)$ is a $V$-mean graph.

Proof. Let $G=C_{n}(3,1)$. Let $V(G)=\left\{v_{1}, v_{2}, \ldots, v_{n}\right\}$ and $E(G)=$ $\left\{v_{1} v_{3}, v_{n} v_{1}, v_{i} v_{i+1}: 1 \leq i \leq n-1\right\}$. Then $G$ has order $n$ and size $n+1$. Let $r=\left\lceil\frac{n}{2}\right\rceil$. The edges of $G$ are assigned labels as follows: Define $f: E(G) \longrightarrow\{0,1,2, \ldots, n+1\}$ as follows : The integers $0,1,2,3$ are respectively assigned to the edges $v_{1} v_{2}, v_{2} v_{3}, v_{1} v_{3}$, and $v_{n} v_{1}$. The odd and even integers of $\{4,5,6, \ldots, n\}$ are respectively arranged in increasing sequences $\alpha_{1}, \alpha_{2}, \ldots, \alpha_{r-2}$ and $\beta_{1}, \beta_{2}, \ldots, \beta_{n-r-1}$ and $\alpha_{k}$ is assigned to $v_{k+2} v_{k+3}$, and $\beta_{k}$ is assigned to $v_{n-k} v_{n-k+1}$. 


\begin{tabular}{|c|c|}
\hline Vertex & Induced edge label \\
\hline$v_{1}$ & 2 \\
\hline$v_{2}$ & 1 \\
\hline$v_{3}$ & 3 \\
\hline$v_{n}$ & $\beta_{1}=4$ \\
\hline$v_{k+3}, 1 \leq k \leq n-r-2$ & $\beta_{k+1}$ \\
\hline$v_{n-k}, 1 \leq k \leq r-2$ & $\alpha_{k}$ \\
\hline
\end{tabular}

Table 2. Induced vertex labels

Clearly the assignment is an injective function and the set of induced vertex labels is $\{1,2, \ldots, n\}$, as illustrated in Table 2 . Thus $G$ is a $V$-mean graph. $\square V$-mean labeling of $C_{8}(3,1)$ and $C_{9}(3,1)$ are shown in Figure 4 .
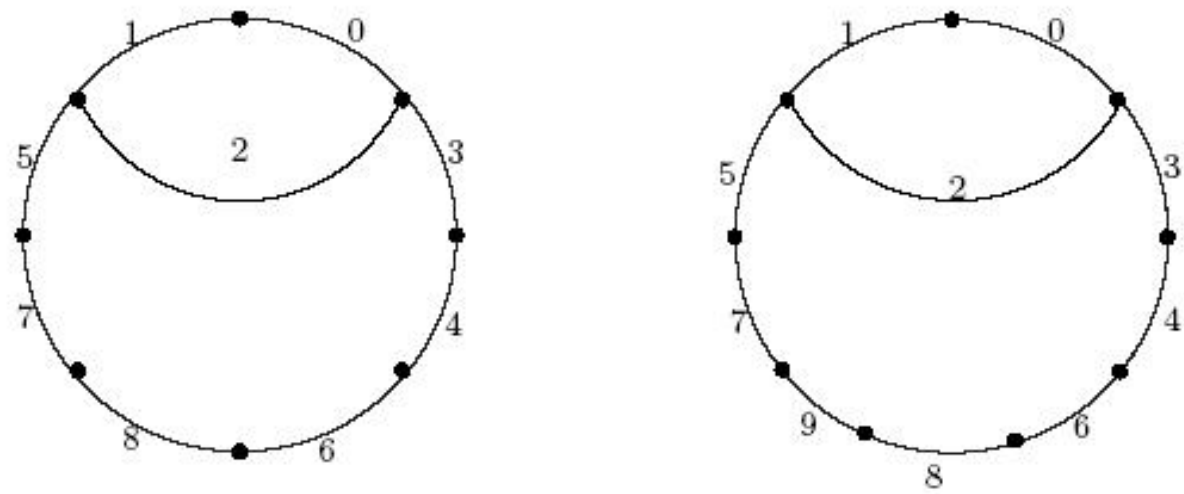

FigURE 4. $V$-mean labeling of $C_{n}(3,1)$ for $n=8,9$.

Theorem 2.5. If $m \in\{n, n+1, n+2\}$, the graph obtained from the two cycles $C_{n}$ and $C_{m}$ by adding a new edge joining a vertex of $C_{n}$ and $C_{m}$ is a $V$-mean graph.

Proof. Let $G$ be the graph consisting of two cycles $C_{n}: v_{1} v_{2} \ldots v_{n}$ and $C_{m}: u_{1} u_{2} \ldots u_{m}$ and $e_{0}=v_{n} u_{1}$ be the bridge connecting them. Then $G$ has 
order $m+n$ and size $m+n+1$. Let $e_{i}=v_{i} v_{i+1}, 1 \leq i \leq n-1$ and $e_{n}=v_{n} v_{1}$ and $e_{i}^{\prime}=u_{i} u_{i+1}, 1 \leq i \leq m-1$, and $e_{m}^{\prime}=u_{m} u_{1}$. Let $r=\left\lceil\frac{n}{2}\right\rceil$. Define $f: E(G) \longrightarrow\{0,1,2, \ldots, m+n+1\}$ as follows:

$$
\begin{gathered}
f\left(e_{i}\right)= \begin{cases}1 & \text { if } i=0 \\
2 i+1 & \text { if } 1 \leq i \leq r-1 \\
2(n-i) & \text { if } r \leq i \leq n\end{cases} \\
f\left(e_{i}^{\prime}\right)=n+i \text { if } 1 \leq i \leq m .
\end{gathered}
$$

Then

$$
\begin{gathered}
f^{V}\left(v_{i}\right)= \begin{cases}2 i & \text { if } 1 \leq i \leq r-1 \\
2(n-i)+1 & \text { if } r+1 \leq i \leq n \\
2(n-i) & \text { if } n \text { is even and } i=r \\
2(n-i)+1 & \text { if } n \text { is odd and } i=r\end{cases} \\
f^{V}\left(u_{i}\right)=n+i \text { if } 1 \leq i \leq m .
\end{gathered}
$$

Clearly $f$ is an injective function and the set of induced vertex labels is $\{1,2, \ldots, n+m\}$. Hence the theorem.

A $V$-mean labeling of the graph obtained from $C_{7}$ and $C_{9}$ is shown in Figure 5.

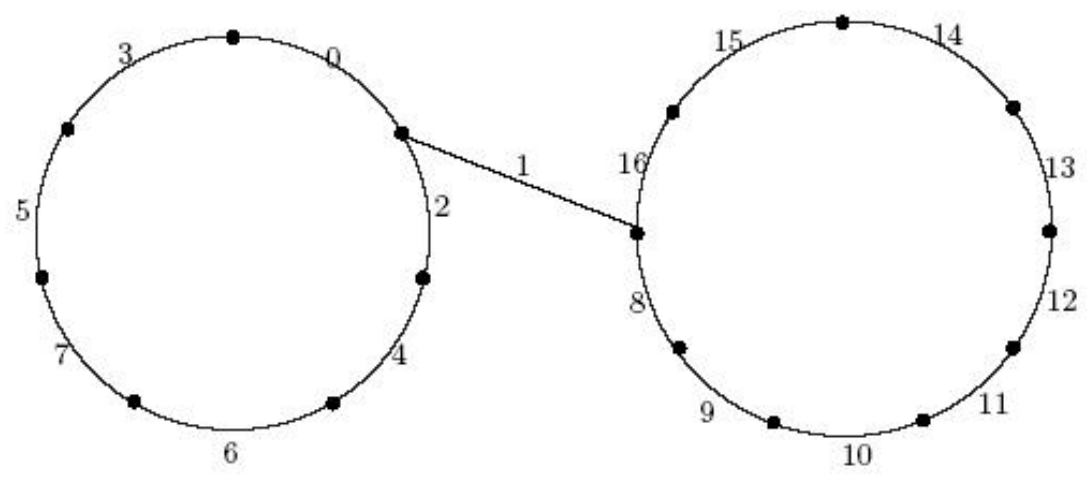

FIgURE 5. A $V$-mean labeling of a graph obtained from $C_{7}$ and $C_{9}$

Theorem 2.6. The graph $C_{n} \cup C_{m}$ is a $V$-mean graph. 
Proof. Let $\left\{e_{1}, e_{2}, \ldots, e_{n}\right\}$ be the edge set of $C_{n}$ such that $e_{i}=v_{i} v_{i+1}$, $1 \leq i \leq n-1, e_{n}=v_{n} v_{1}$ and $\left\{e_{1}^{\prime}, e_{2}^{\prime}, \ldots, e_{m}^{\prime}\right\}$ be the edge set of $C_{m}$ such that $e_{i}^{\prime}=u_{i} u_{i+1}, 1 \leq i \leq m-1, e_{m}^{\prime}=u_{m} u_{1}$. Then the graph $G=C_{n} \cup C_{m}$ has order and size both equal to $m+n$. Let $m \geq n$. Define $f: E(G) \longrightarrow\{0,1,2, \ldots, m+n\}$ as follows:

$$
\begin{gathered}
f\left(e_{i}\right)= \begin{cases}i-1 & \text { if } 1 \leq i \leq\left\lfloor\frac{n}{2}\right\rfloor \\
i & \text { if }\left\lfloor\frac{n}{2}\right\rfloor+1 \leq i \leq n-1, \\
n+1 & \text { if } i=n\end{cases} \\
f\left(e_{i}^{\prime}\right)=\left\{\begin{array}{ll}
n+2 i+1 & \text { if } 1 \leq i \leq\left\lceil\frac{m}{2}\right\rceil-1 \\
n+2(m-i) & \text { if }\left\lceil\frac{m}{2}\right\rceil \leq i \leq m
\end{array} .\right.
\end{gathered}
$$

Then

$$
\begin{gathered}
f^{V}\left(v_{i}\right)= \begin{cases}\left\lfloor\frac{n}{2}\right\rfloor+1 & \text { if } i=1 \\
i-1 & \text { if } 2 \leq i \leq\left\lfloor\frac{n}{2}\right\rfloor+1 \\
i & \text { if }\left\lfloor\frac{n}{2}\right\rfloor+2 \leq i \leq n\end{cases} \\
f^{V}\left(u_{i}\right)=\left\{\begin{array}{ll}
n+2 i & \text { if } 1 \leq i \leq\left\lfloor\frac{m}{2}\right\rfloor \\
n+2(m-i)+1 & \text { if }\left\lfloor\frac{m}{2}\right\rfloor+1 \leq i \leq m
\end{array} .\right.
\end{gathered}
$$

Clearly $f$ is an injective function and the set of induced vertex labels is $\{1,2, \ldots, n+m\}$. Hence the theorem.

A $V$-mean labeling of $C_{8} \cup C_{12}$ is shown in Figure 6 . 


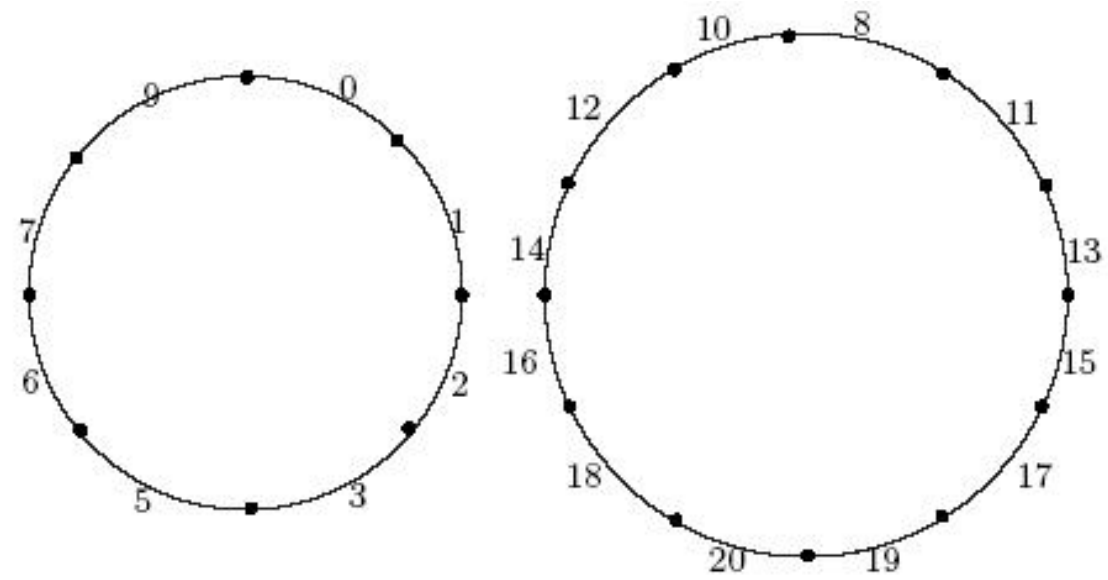

Figure 6. A $V$-mean labeling of $C_{8} \cup C_{12}$

Theorem 2.7. If $m \in\{3,4\}$, the graph $G$ obtained by identifying one vertex of the cycle $C_{m}$ with a vertex of $C_{n}$ is a $V$-mean graph.

\section{Proof.}

case $1 m=3$.

Let $G$ be the graph consisting of two cycles $C_{3}: v_{1} v_{2} v_{3} v_{1}$ and $C_{n}$ : $v_{3} v_{4} \ldots v_{n+2} v_{3}$. Let $r=\left\lceil\frac{n}{2}\right\rceil$. Define $f: E(G) \longrightarrow\{0,1,2, \ldots, n+3\}$ as follows: The integers $0,1,2,3,4$ are assigned respectively to the edges $v_{1} v_{2}, v_{3} v_{1}, v_{n+2} v_{3}, v_{2} v_{3}, v_{3} v_{4}$. The odd and even integers of $\{5,6,7, \ldots, n+2\}$ are arranged respectively in increasing sequences $\alpha_{1}, \alpha_{2}, \ldots, \alpha_{r-1}$ and $\beta_{1}, \beta_{2}, \ldots, \beta_{n-r-1}$ and $\alpha_{k}$ is assigned to $v_{k+3} v_{k+4}$, and $\beta_{k}$ is assigned to $v_{n+2-k} v_{n+3-k}$. 


\begin{tabular}{|c|c|}
\hline Vertex & vertex label \\
\hline$v_{k}, k=1,2,3$ & $k$ \\
\hline$v_{n+2}$ & 4 \\
\hline$v_{4}$ & $\alpha_{1}(=5)$ \\
\hline$v_{k+4}, 1 \leq k \leq n-r-1$ & $\beta_{k}$ \\
\hline$v_{n-k+2}, 1 \leq k \leq r-2$ & $\alpha_{k+1}$ \\
\hline
\end{tabular}

Table 3. Induced vertex labels

\begin{tabular}{|c|c|}
\hline Vertex & vertex label \\
\hline$v_{1}$ & 3 \\
\hline$v_{2}$ & 1 \\
\hline$v_{3}$ & 2 \\
\hline$v_{4}$ & 4 \\
\hline$v_{5}$ & 5 \\
\hline$v_{n+3}$ & 6 \\
\hline$v_{6}$ & $\alpha_{1}(=7)$ \\
\hline$v_{k+6}, 1 \leq k \leq n-r-2$ & $\beta_{k}$ \\
\hline$v_{n-k+3}, 1 \leq k \leq r-2$ & $\alpha_{k+1}$ \\
\hline
\end{tabular}

Table 4. Induced vertex labels

Clearly the assignment is an injective function and the set of induced vertex labels is $\{1,2, \ldots, n+2\}$, as illustrated in Table 3 . Thus $G$ is a $V$-mean graph.

case $2 m=4$.

Let $G$ be the graph consisting of two cycles $C_{4}: v_{1} v_{2} v_{3} v_{4} v_{1}$ and $C_{n}$ : $v_{4} v_{5} \ldots v_{n+3} v_{4}$. Let $r=\left\lfloor\frac{n}{2}\right\rfloor$. Define $f: E(G) \longrightarrow\{0,1,2, \ldots, n+4\}$ as follows: The integers $0,1,2,3,4,5,6$ are assigned respectively to the edges $v_{1} v_{2}, v_{2} v_{3}, v_{3} v_{4}, v_{n+3} v_{4}, v_{4} v_{5}, v_{4} v_{1}, v_{5} v_{6}$. The odd and even integers of $\{7,8, \ldots, n+3\}$ are arranged respectively in increasing sequences $\alpha_{1}, \alpha_{2}, \ldots, \alpha_{r-1}$ and $\beta_{1}, \beta_{2}, \ldots, \beta_{n-r-2}$ and $\alpha_{k}$ is assigned to $v_{k+5} v_{k+6}$, and $\beta_{k}$ is assigned to $v_{n+3-k} v_{n+4-k}$. 
Clearly the assignment is an injective function and the set of induced vertex labels is $\{1,2, \ldots, n+3\}$, as illustrated in Table 4 . Thus $G$ is a $V$-mean graph.

Fox example $V$-mean labeling of graphs obtained from $C_{3}$ and $C_{8}$ and $C_{4}$ and $C_{8}$ are shown in Figure 7.
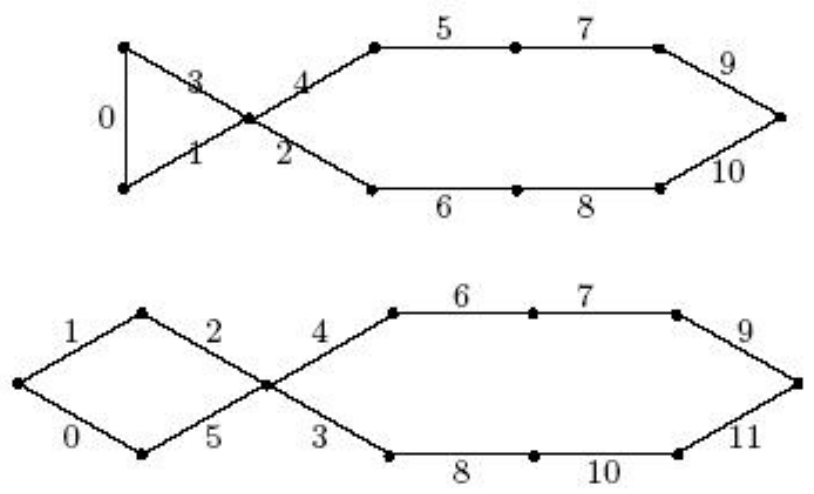

FIGURE 7 .

\section{Some disconnected V-mean graphs}

In this section we present a method to construct disconnected V-mean graphs from $V$-mean graphs. The following observation is obvious from the definition of $V$-mean labeling.

Observation 3.1. If $f$ is any $V$-mean labeling of a $(p, q)$ graph $G$, then $f(e) \geq p$ for some edge $e \in E(G)$. In particular, if $p \geq q$ then $f(e) \leq p$ for every edge $e \in E(G)$ and hence $f(e)=p$ for some edge $e \in E(G)$.

Notation 3.2. We call a $V$-mean labeling $f$ of a graph $G(p, q)$ as type- $A$, if $f(e) \leq p$ for every edge $e \in E(G)$, type- $B$ if $f(e) \geq 1$ for every edge $e \in E(G)$, and type- $A B$ if $1 \leq f(e) \leq p$ for every edge $e \in E(G)$. For $S \in\{A, B, A B\}$, we call $G$ as $V$-mean graph of type- $S$ if it has a $V$-mean labeling $f$ of type- $S$.

Remark 3.3. We observe that the $V$-mean graphs presented in [3] and Theorem 2.1 through Theorem 2.7 can be classified as given in Table 5 . 


\begin{tabular}{|c|l|c|}
\hline S.N0 & $V$-mean Graph & Type \\
\hline 1 & $C_{n}$ & $A$ \\
\hline 2 & $C_{n} \odot K_{1}^{C}$ & $A$ \\
\hline 3 & $C_{n}(3,1)$ & $A$ \\
\hline 4 & $\begin{array}{l}\text { The graph consisting of two cycles } C_{n} \text { and } C_{m} \\
\text { connected by a bridge }\end{array}$ & $A$ \\
\hline 5 & The graph $C_{n} \cup C_{m}$ where $m \in\{n, n+1, n+2\}$ & $A$ \\
\hline 6 & $\begin{array}{l}\text { The graph obtained by identifying one vertex of } \\
\text { the cycle } C_{m} \text { with a vertex of } C_{n} \text { when } m=3 \text { or } 4\end{array}$ & $A B$ \\
\hline 7 & $P_{n}$ where $n \geq 3$ & $A B$ \\
\hline 8 & $P_{n} \odot K_{m}^{C}$ where $n \geq 2$ & $A B$ \\
\hline 9 & $K_{1, n}$ if and only if $n \equiv 0(m o d 2)$ & $A B$ \\
\hline 10 & $\begin{array}{l}\text { The graph } S\left(K_{1, n}\right), \text { obtained by } \\
\text { subdividing every edge of } K_{1, n}\end{array}$ & $A B$ \\
\hline 11 & Dragon graph & $\begin{array}{l}\text { The graph obtained by identifying one vertex of cycle } \\
C_{m} \text { with the central vertex of } K_{1, n} \text { when } m=3 \text { or } 4\end{array}$ \\
\hline 12 & \multicolumn{2}{|l}{. } \\
\hline
\end{tabular}

Table 5. V-mean graphs

Let $f$ be a $\mathrm{V}$-mean labeling of $G\left(p_{1}, q_{1}\right)$ and $g$ be a $V$-mean labeling of $H\left(p_{2}, q_{2}\right)$. Observe that the graph $G \cup H$ has order $p=p_{1}+p_{2}$ and size $q=q_{1}+q_{2}$. Define $h: E(G \cup H) \longrightarrow\left\{0,1,2, \ldots, q_{*}\right\}$ as follows:

$$
h(e)=\left\{\begin{array}{ll}
f(e) & \text { if } e \in E(G) \\
g(e)+p_{1} & \text { if } e \in E(H)
\end{array} .\right.
$$

Suppose $f$ is of type- $A$ and $g$ is of type- $B$. Then $f(e) \leq p_{1}$ for every edge $e \in E(G)$ and $g(e) \geq 1$ for every edge $e \in E(H)$. As $f$ and $g$ are injective functions, $f(e) \leq p_{1}$ for every edge $e \in E(G)$ and $g(e)+p_{1} \geq p_{1}+1$ for every edge $e \in E(H), h$ is injective.

Suppose $f$ is of type- $A$ and $g$ is of type- $A B$. Then $f(e) \leq p_{1}$ for every edge $e \in E(G)$ and $1 \leq g(e) \leq p_{2}$ for every edge $e \in E(H)$. As $f$ and $g$ are injective functions, $f(e) \leq p_{1}$ for every edge $e \in E(G)$ and $p_{1}+1 \leq g(e)+p_{1} \leq p_{1}+p_{2}$ for every edge $e \in E(H), h$ is injective and $h(e) \leq p$ for every edge $e \in E(G \cup H)$.

Suppose $f$ is of type- $A B$ and $g$ is of type- $B$. Then $1 \leq f(e) \leq p_{1}$ for every edge $e \in E(G)$ and $g(e) \geq 1$ for every edge $e \in E(H)$. As $f$ and $g$ are injective functions, $1 \leq f(e) \leq p_{1}$ for every edge $e \in E(G)$ and 
$p_{1}+1 \leq g(e)+p_{1}$ for every edge $e \in E(H), h$ is injective and $h(e) \geq 1$ for every edge $e \in E(G \cup H)$.

Suppose, both $f$ and $g$ are of type- $A B$. Then $1 \leq f(e) \leq p_{1}$ for every edge $e \in E(G)$ and $1 \leq g(e) \leq p_{2}$ for every edge $e \in E(H)$. As, $f$ and $g$ are injective functions, $1 \leq f(e) \leq p_{1}$ for every edge $e \in E(G)$ and $p_{1}+1 \leq g(e)+p_{1} \leq p_{1}+p_{2}$ for every edge $e \in E(H), h$ is injective and $1 \leq h(e) \leq p$ for every edge $e \in E(G \cup H)$.

The set of induced vertex labels of $G \cup H$ in all four cases is as follows:

$$
\begin{gathered}
h^{V}(V(G \cup H))=\left\{f^{V}(v): v \in V(G)\right\} \cup\left\{p_{1}+g^{V}(u): u \in V(H)\right\} \\
=\left\{1,2, \ldots, p_{1}\right\} \cup\left\{p_{1}+1, p_{1}+2, \ldots, p_{1}+p_{2}\right\} \\
=\left\{1,2, \ldots, p_{1}+p_{2}\right\} .
\end{gathered}
$$

Thus we have the following four theorems.

Theorem 3.4. If $G\left(p_{1}, q_{1}\right)$ is a $V$-mean graph of type- $A$ and $H\left(p_{2}, q_{2}\right)$ is a $V$-mean graph of type- $B$, then $G \cup H$ is $V$-mean.

Theorem 3.5. If $G\left(p_{1}, q_{1}\right)$ is a $V$-mean graph of type- $A$ and $H\left(p_{2}, q_{2}\right)$ is a $V$-mean graph of type- $A B$, then $G \cup H$ is $V$-mean graph of type- $A$.

Theorem 3.6. If $G\left(p_{1}, q_{1}\right)$ is a $V$-mean graph of type- $A B$ and $H\left(p_{2}, q_{2}\right)$ is a $V$-mean graph of type- $B$, then $G \cup H$ is $V$-mean graph of type- $B$.

Theorem 3.7. If both $G\left(p_{1}, q_{1}\right)$ and $H\left(p_{2}, q_{2}\right)$ are $V$-mean graphs of type$A B$ then the graph $G \cup H$ is $V$-mean graph of type- $A B$.

Corollary 3.8. Let $G$ be a tree or a unicyclic graph or a two regular graph. If $G$ is $V$-mean and $H$ is a $V$-mean graph of type- $B$, then $G \cup H$ is $V$-mean.

Corollary 3.9. If $G(p, q)$ is $V$-mean graph of type- $A B$ then, the graph $m G$ is $V$-mean graph type- $A B$.

Corollary 3.10. If both $G\left(p_{1}, q_{1}\right)$ and $H\left(p_{2}, q_{2}\right)$ are $V$-mean graphs of type- $A B$, then the graph $m G \cup n H$ is $V$-mean graph of type- $A B$.

Corollary 3.11. If $G\left(p_{1}, q_{1}\right)$ is a $V$-mean graph of type- $A$ and $H\left(p_{2}, q_{2}\right)$ is a $V$-mean graph of type- $A B$, then $G \cup m H$ is $V$-mean graph of type- $A$. 
It is interesting to note that a number of disconnected $V$-mean graphs can be obtained by applying Theorem 3.4 through Corollary 3.11 on $V$ mean graphs listed in Table 5. For example, the graph $\bigcup_{i=1}^{k} P_{n_{i}}$, where $n_{i} \geq 3$, the graph $m P_{n}$ where $n \geq 3$, the graph $C_{n} \cup\left(\bigcup_{i=1}^{k} P_{n_{i}}\right)$, where

$n_{i} \geq 3$, the graph $C_{n} \cup k P_{m}$ where $m \geq 3$, the graph $C_{n} \cup C_{m} \cup\left(\bigcup_{i=1}^{k} P_{n_{i}}\right)$, where $n_{i} \geq 3$, the graph $C_{n} \cup C_{m} \cup k P_{t}$ where $t \geq 3$ are some of such graphs. To illustrate this a $\mathrm{V}$-mean labeling of $C_{10} \cup P_{4} \cup P_{5}$ and a $\mathrm{V}$-mean labeling of $\left(C_{8} \cup C_{12}\right) \cup K_{1,8}$ are given in Figure 8 and Figure 9 respectively.

\section{References}

[1] B. D. Acharya and K. A. Germina, Vertex-graceful graphs, Journal of Discrete Mathematical Science \& Cryptography, Vol. 13, No. 5, pp. 453-463, (2010).

[2] J. A. Gallian. A dynamic survey of graph labeling. The electronic journal of combinatorics, 18, (2011) \#DS6.

[3] A. Lourdusamy and M. Seenivasan. Vertex-mean graphs. International journal of Mathematical Combinatorics, 3, pp. 114-120, (2011).

[4] S. Somasundaram and R. Ponraj, Mean labelings of graphs, Natl Acad, Sci. Let., 26, pp. 210-213, (2003).

\section{A. Lourdusamy}

Department of Mathematics,

St.Xavier's College (Autonomous),

Palayamkottai, Tirunelveli,

India

e-mail : lourdugnanam@hotmail.com

and

\section{Seenivasan}

Department of Mathematics,

Sri Paramakalyani College,

Alwarkurichi-627412, Tirunelveli,

India

e-mail : msvasan_22@yahoo.com 